\title{
A Rhodamine-Based Probe for Detection of Nerve Agents Simulants
}

\author{
Liang ZONG ${ }^{\mathrm{a}}$, Jingning $\mathrm{ZHANG}^{\mathrm{b}}$, Dan $\mathrm{LI}^{\mathrm{a}}$, Shaohui SUI ${ }^{\mathrm{a}}$, Yanhua XIAO ${ }^{\mathrm{a}}$, Jian $\mathrm{LI}^{\mathrm{a}}$, \\ Meng LIU ${ }^{\mathrm{a}}$, Bo ZHUANG ${ }^{\mathrm{a}}$, Daxue $\mathrm{LI}^{\mathrm{a}}$ and Weihui WU,1 \\ ${ }^{a}$ Department of Chemical Defence, Institute of Chemical Defence, Beijing 102205, \\ China \\ ${ }^{\mathrm{b}}$ PLA Unit No. 66114, Beijing 100086, China
}

\begin{abstract}
In this paper, a rhodamine-based fluorescent and chromogenic probe, N(Rhodamine B)-lactam-2-aminobenzyl alcohol (RB-AB), was designed to detect nerve agent simulants. We firstly synthesized RB-AB probe by using rhodamine $\mathrm{B}$ and 2-aminobenzyl alcohol as main materials. Secondly, the RB-AB probe was applied to evaluate its ability to detect two nerve agent simulants, diethyl chloride phosphate (DCP) and methyl ethyl chloride phosphate (MECP). It was assumed that $\mathrm{RB}-\mathrm{AB}$ could react with the nerve agent simulants through the benzyl alcohol group and then undergoes structural changes. As a result, the RB-AB detection solution shows fluorescent and color changes during detecting process. The maximum intensity of fluorescence emission increases with the addition of DCP or MECP in a dose-dependent manner. The LOD (limit of detection) of the probe is about 20 ppm for DCP. Moreover, a significant pink color change can be observed in the RB$\mathrm{AB}$ system within a few seconds when detecting DCP or MECP. In conclusion, a rhodamine-based molecule as a fluorescent and chromogenic probe was developed for detecting nerve agent simulants. The RB-AB probe solutions can give rapid and off-on type optical changes including color and fluorescence when reacting with DCP or MECP. We anticipate that RB-AB probe can be used as a helpful tool for visual and fluorescent detection of nerve agents when meeting with terrorist attacks involving with these agents so that effective measures could be promptly taken to cope with the crises.
\end{abstract}

Keywords. Rhodamine-based probe, diethyl chloride phosphate, methyl ethyl chloride phosphate

\section{Introduction}

Nerve agents are a category of organophosphorus compounds with very high inhibitory activity to the enzyme of acetylcholinesterase in nerve system of the body [1-3]. VX, GD (Soman), GB (Sarin), GA (Tabun) (scheme 1a) are the typical representatives among them [4-6]. As a kind of highly toxic chemical warfare agents, nerve agents have shown their great threats to human beings in military conflicts. In recent years, increasing terrorist activities involving nerve agents have been earning wide concerns from the public and society. Motivated by the urgent requirement of early warning in a nerve agent terrorist attack, researchers have been dedicated to the development of sensitive and convenient techniques for nerve agent detection [7-13]. Many different kinds of methods

\footnotetext{
${ }^{1}$ Corresponding Author, Weihui WU, Department of Chemical Defence, Institute of Chemical Defence,
} Beijing 102205, China; E-mail: wuwh2012@163.com. 
have been developed for nerve agent detection including mass spectrometry, infrared spectroscopy, capillary electrophoresis, etc. However, most of the methods are usually time-absorbing and dependent on large and expensive instruments with complex operation, which greatly limits their practical application in nerve agent detection. Thus, it is highly urgent to explore a portable, rapid and reliable approach for detecting nerve agents.

Fluorescent probes provide a handy and simple technique of detection as they can generate fluorescence changes (fluorescence intensity, lifetime, excitation or emission spectra, for instance) when fluorescent probes react with nerve agents [12, 14-20]. Fluorescence probe detection only requires simple and portable devices for detection of target analytes with high sensitivity and selectivity, which can make it playing an increasingly important role in nerve agent on-site detection.

Previously [16], we reported a fluorescent probe, N-(Rhodamine B)-lactam-2aminoethanol (RB-AE), for detecting a nerve agent simulant of DCP (scheme 1b). It was proposed that RB-AE undergoes phosphoester and oxazoline formation when it reacts with DCP, which eventually leads to spirolactam-ring opening. As a result, the species with high fluorescence and clear color is hence formed, which can be detected by simple instruments and even by naked eye because unreacted the RB-AE with intramolecular spirolactam ring is nonfluorescent and colorless.

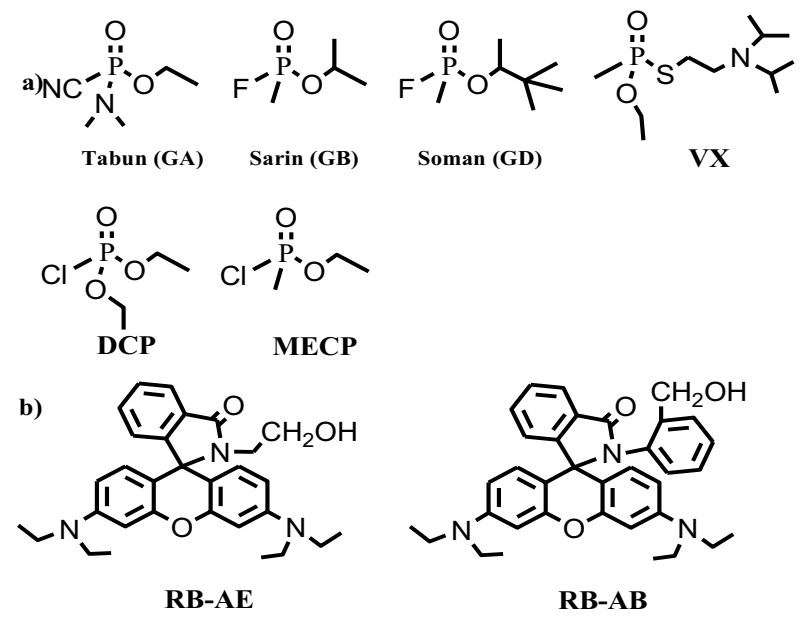

Scheme 1. (a) Molecular structures of several typical nerve agents, simulants and (b) two fluorescent and chromogenic probes, RB-AE in our previous work [16] and RB-AB in this work.

Herein, we presented another fluorescent and chromogenic probe, $\mathrm{N}$-(Rhodamine B)-lactam-2-aminobenzyl alcohol (RB-AB, scheme 1), for detecting nerve agent simulants. RB-AE and RB-AB share similar structure and functional groups, thus showing similar reactivity for detection. RB-AE reacts with target analytes through $2-$ aminoethanol group, while RB-AB does it through 2-aminobenzyl alcohol group. We reasoned that the benzyl alcohol group in RB-AB is also an excellent nucleophilic function group that can attack on electrophilic target molecule, leading to formation of phosphate ester. The phosphate ester intermediate of RB-AB could then generate detectable fluorescence and color species through intramolecular structural transformation, which is similar to the supposed mechanism of RB-AE for detection. 


\section{General Methods}

Most of the chemical agents and organic solvents were purchased from Aladdin Company. The product in each step of organic synthesis was separated by silica gel Column chromatography. ${ }^{1} \mathrm{H}$ NMR and ${ }^{13} \mathrm{C}$ NMR were measured on a conventional NMR spectrometer (JEOL JNM-ECS $400 \mathrm{MHz}$ ). ESI-MS measurements were performed by an Angilent 6460 mass spectrometer. The fluorescence data were collected by a fluorescence spectrophotometer (Hitachi F-4600) at RT. Fluorescence and UV-Vis absorption spectra were collected according to standard procedures.

\section{Synthesis Procedure}

\subsection{Preparation of Compound 1}

A mixture of rhodamine $\mathrm{B} 6 \mathrm{~g}(13.54 \mathrm{mmol})$ and $30 \% \mathrm{NaOH}$ solution $(100 \mathrm{~mL})$ was stirred at RT for 4 hours. The cooling solution was extracted with $\mathrm{CH}_{3} \mathrm{COOEt}$, and then the $\mathrm{CH}_{3} \mathrm{COOEt}$ extract was washed with $\mathrm{NaHCO}_{3}$ solution. The resulting resolution was dried with $\mathrm{Na}_{2} \mathrm{SO}_{4}$ overnight. Compound 1 was obtained after $\mathrm{CH}_{3} \mathrm{COOEt}$ was further removed under vacuo. Yield: $4.093 \mathrm{~g}(68.23 \%)$.

\subsection{Preparation of Compound 2}

Compound $10.63 \mathrm{~g}(1.43 \mathrm{mmol})$ was added in dry DCM (dichloromethane), and the solution was stirred for several minutes. Then add $3.79 \mathrm{~mL}$ phosphorus oxychloride $\left(\mathrm{POCl}_{3}\right)$ to the solution. The mixture was then heated and refluxed. After refluxed for 4 hours, the solvent was cooling ad removed. The obtained residue, namely compound 2 , was directly used in the step of compound 3 preparation.

\subsection{Preparation of Compound 3}

The solution of $2.72 \mathrm{~g}$ Tertbutyldimethylsilyl chloride (TBSCl) in $20 \mathrm{~mL}$ pyridine was added to $35 \mathrm{~mL}$ pyridine solution containing 2-aminobenzyl alcohol. The mixed solution was kept at $60-70^{\circ} \mathrm{C}$ for 1 hour. The solvent was evaporated after cooling, and the solvent of $\mathrm{CH}_{3} \mathrm{COOEt}_{2}$ was added to the obtained residue. The $\mathrm{CH}_{3} \mathrm{COOEt}_{2}$ solution was washed with water, saturated citric acid solution and saturated $\mathrm{NaCl}$ solution separately. The washed $\mathrm{CH}_{3} \mathrm{COOEt}_{2}$ solution was dried with $\mathrm{Na}_{2} \mathrm{SO}_{4}$ overnight. The dried solution was further evaporated under vacuo and the obtained crude product was purified to give compound 3. Yield: $0.76 \mathrm{~g}(21.23 \%)$.

\subsection{Preparation of Probe Compound 4}

Compound $30.758 \mathrm{~g}$ and triethylamine $\left(\mathrm{Et}_{3} \mathrm{~N} 4 \mathrm{~mL}\right)$ were separately added in $30 \mathrm{~mL}$ dry tetrahydrofuran (THF) under $\mathrm{N}_{2}$ atmosphere. The compound 2 was then added dropwise. The mixed solution stayed at RT for 18 hours, then heated at $45^{\circ} \mathrm{C}$ for another 2 hours. After cooling, the mixture was dissolved with $\mathrm{CH}_{3} \mathrm{COOEt}_{2}$. The $\mathrm{CH}_{3} \mathrm{COOEt}_{2}$ solution was separately washed with water and saturated $\mathrm{NaCl}$ solution. The resulting solution 
was dried with $\mathrm{Na}_{2} \mathrm{SO}_{4}$ overnight. The dried solution was further evaporated and the resultant residue was finally purified to produce compound 4 . Yield: $0.87 \mathrm{~g} \mathrm{(40.91 \% ).}$

\subsection{Preparation of Probe $R B-A B$}

$30 \mathrm{~mL}$ THF was added to compound $40.87 \mathrm{~g}(1.31 \mathrm{mmol})$ in a round bottom flask. After dissolving, $2.03 \mathrm{~g}$ tetrabutylammonium fluoride (TBAF) was added. The solution stayed at RT for an half of hour, and then $80 \mathrm{~g}$ ice was added. The solution was extracted with $\mathrm{CH}_{3} \mathrm{COOEt}_{2}$ for 3 times. The $\mathrm{CH}_{3} \mathrm{COOEt}_{2}$ extract was separately washed with water and a saturated $\mathrm{NaCl}$ solution. The resulting solution was dried with $\mathrm{Na}_{2} \mathrm{SO}_{4}$ overnight. The probe RB-AB was obtained after the solvent was removed by rotary evaporation and the residue was further purified. Yield: $0.595 \mathrm{~g}(82.98 \%)$.

${ }^{1} \mathrm{H}-\mathrm{NMR}\left(400 \mathrm{MHz}, \mathrm{DMSO}-\mathrm{d}_{6}\right): \delta$ 7.89-7.91(m, 1H), 7.64-7.66 (m, 2H), 7.38-7.42 $(\mathrm{m}, 1 \mathrm{H}), 7.20-7.24(\mathrm{t}, 2 \mathrm{H}), 6.87-6.91(\mathrm{t}, 1 \mathrm{H}), 6.47-6.49(\mathrm{~m}, 3 \mathrm{H}), 6.30(\mathrm{~d}, 1 \mathrm{H}), 6.27(\mathrm{~s}, 1 \mathrm{H})$, $6.13(\mathrm{~s}, 1 \mathrm{H}), 5.91-5.93(\mathrm{~d}, 1 \mathrm{H}), 4.84(\mathrm{~s}, 1 \mathrm{H}), 4.24-4.28(\mathrm{~d}, 1 \mathrm{H}), 3.63-3.67(\mathrm{~d}, 1 \mathrm{H}), 3.26-$ 3.45 (m), 1.04-1.11 (t, 12H); ${ }^{13} \mathrm{C}-\mathrm{NMR}$ (400 MHz, DMSO-d 6 ): $\delta 165.64,154.15,153.91$, $151.85,148.27,142.05,133.90,132.93,131.83,129.42$, 129.10, 128.92, 128.24, 127.71, $126.89,126.67,124.95,123.67,109.19,108.15,107.27,105.87,98.22,97.25,68.14$, 59.45, 44.46, 44.05, 12.87 ppm; ESI-MS $\left(\mathrm{C}_{35} \mathrm{H}_{37} \mathrm{~N}_{3} \mathrm{O}_{3}\right)$ : calculated $(\mathrm{M}+\mathrm{H})^{+}:$: 548.28, found: 548.44 .

\section{Results and Discussion}

\subsection{Molecule Design and Synthesis of Probe RB-AB}

Among the methods for detecting nerve agents, the utilization of fluorescent probes exhibits their merits of simplicity and convenience because they can produce fluorescence changes during the process of detection. A reactive probe molecule should have the property of reacting with nerve agents or their simulants, and the resultant product should have obvious fluorescence changes compared with the molecule. According to this strategy, Probe RB-AB was designed as the secondary generation of RB-AE for detecting nerve agents. 2-aminobenzyl alcohol was also introduced into the Rhodamine B structure. We anticipated that benzyl alcohol group of RB-AB will undergo nucleophile substitution with nerve agent simulants just like its parent probe of $\mathrm{RB}-\mathrm{AE}$. The resultant phosphate ester of RB-AB will then go through the ring-opening of lactam, which gives obvious fluorescent or optical changes [16].

The Probe RB-AB was routinely synthesized in five steps (see experimental and scheme 2). In the process, all solvents should be dried, and each step of synthesis was performed under a nitrogen atmosphere. The final product of RB-AB was performed by MS and NMR analysis. 

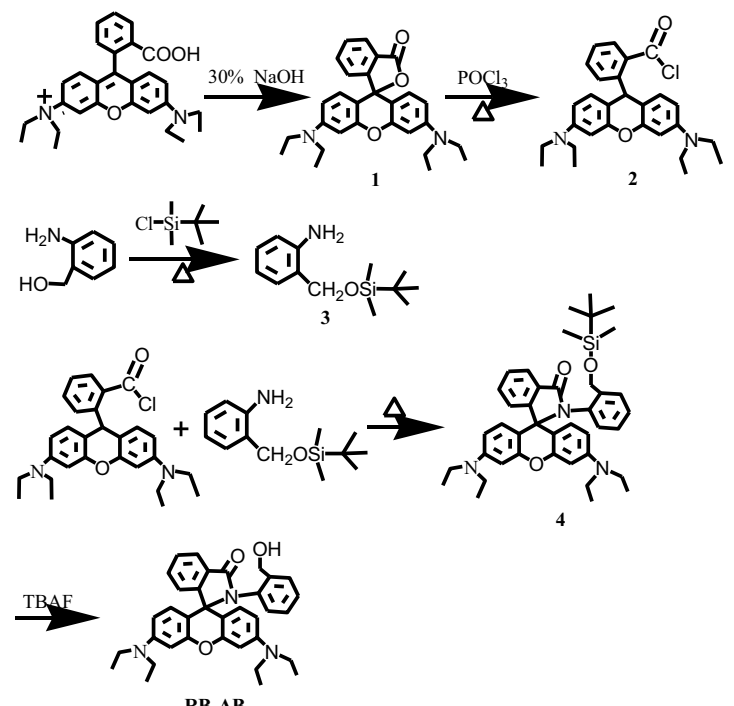

Scheme 2. Synthetic route to probe RB-AB.

\subsection{Detection of DCP and MECP Using Probe RB-AB by Visual and Ultraviolet- Visible Absorption Analysis}

In our study, DCP and DECP were used as the nerve agent simulants to evaluate RBAB's ability to detect because they share similar molecular structure and reactivity to nerve agents $[16,21]$. N, N-dimethyl formamide (DMF) was chosen as the preferred analysis medium due to its excellent solubility of $\mathrm{RB}-\mathrm{AB}$ and a relatively low background value.

To achieve the goal of rapid measurement, $\mathrm{RB}-\mathrm{AB}$ at the concentration of $1 \mathrm{mg} / \mathrm{mL}$ in DMF was applied in our tests as RB-AB's fluorescence and color is inappreciable. Addition of DCP above $50 \mathrm{ppm}$ to RB-AB in DMF quickly develops a pink color (figure 1a), indicating that the RB-AB probe could afford an extremely rapid and easy way to analyze the mimic of nerve agents. Most especially, the pink color becomes deeper and deeper with more and more DCP addition, which affords the potential of semiquantitative sensing the simulant of nerve agents with the naked eye.

Ultraviolet-Visible absorption analysis was then performed. DCP at different concentrations was added to the RB-AB detection solutions, and the spectra were recorded at RT. Figure $1 \mathrm{~b}$ revealed that the characteristic absorption peak of detection solutions was situated around $560 \mathrm{~nm}$, and the peak intensity of absorbance increased continuously when the concentrations of DCP increased from 0 to $250 \mathrm{ppm}$ (figure $1 \mathrm{~b}$ ), which was consistent with the result of color changes.

The similar phenomena were also observed when the stimulant MECP was added to the RB-AB assay solutions. The detection solution became pink from colorless within a couple of seconds when the amount of added MECP was more than $50 \mathrm{ppm}$. The obvious color changes (figure 2) for $\mathrm{RB}-\mathrm{AB}$ reacting with the two nerve agent simulants suggest that $\mathrm{RB}-\mathrm{AB}$ has a potential as a facile and simple tool for detection. 
a)

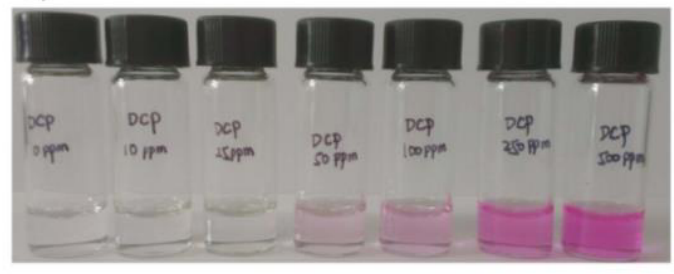

b)

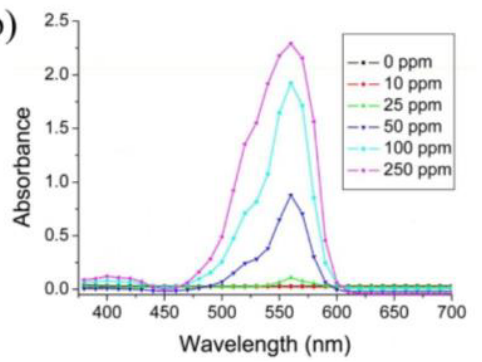

Figure 1. Chromogenic analysis of Probe RB-AB with DCP in DMF solutions: (a) Color changes of Probe RB-AB with DCP at different concentrations (from left to right: 0, 10, 25, 50, 100, 250, and 500 ppm) and (b) UV-Vis spectra of Probe RB-AB with DCP at different concentrations (from bottom to top: 0, 10, 25, 50, 100 and $250 \mathrm{ppm})$.

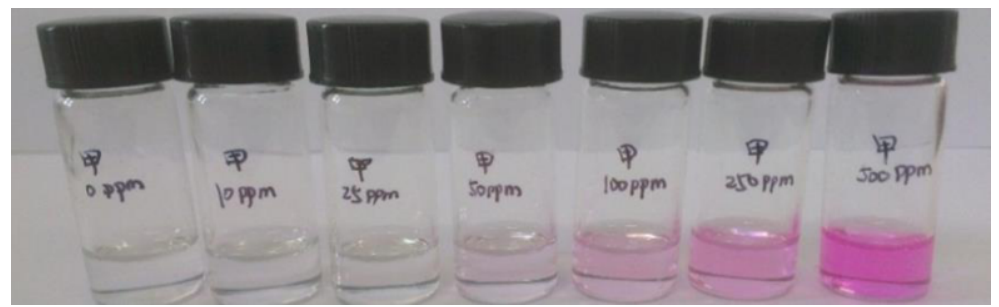

Figure 2. Color changes of Probe RB-AB with MECP at different concentrations (from left to right: $0,10,25$, $50,100,250$, and $500 \mathrm{ppm})$.

To examine the rapidness of $\mathrm{RB}-\mathrm{AB}$ detection system, the color developing time was measured. From table 1, we can see that the response time of Probe RB-AB detection solutions was shortened as the concentration of DCP or MECP increased. It only needed 8 seconds to observe a significant color change when the DCP concentration is $500 \mathrm{ppm}$, which indicated that the Probe RB-AB was fast, simple and suitable for detection of nerve agent mimics.

Table 1. Response time of Probe RB-AB with DCP and MECP in DMF solutions.

\begin{tabular}{lll}
\hline Concentration $\mathbf{( p p m )}$ & DCP $(\mathbf{s})$ & MECP $(\mathbf{s})$ \\
\hline 50 & 60 & 72 \\
100 & 25 & 36 \\
250 & 12 & 16 \\
500 & 8 & 10 \\
\hline
\end{tabular}

\subsection{Detection of DCP and MECP by Probe RB-AB through Fluorescence Spectroscopy}

The optimal excitation and emission wavelength were identified as $520 \mathrm{~nm}$ and $590 \mathrm{~nm}$ separately through preliminary experiments. We monitored the fluorescent intensity changes at different time after DCP was mixed with the RB-AB detection solution. The emission fluorescence intensity of $590 \mathrm{~nm}$ (Ex 520nm) gradually increased with the reaction time extending. An obvious fluorescence emission peak around $590 \mathrm{~nm}$ could be observed within several minutes, indicating that RB-AB could rapidly detect DCP by fluorescence measurement. 

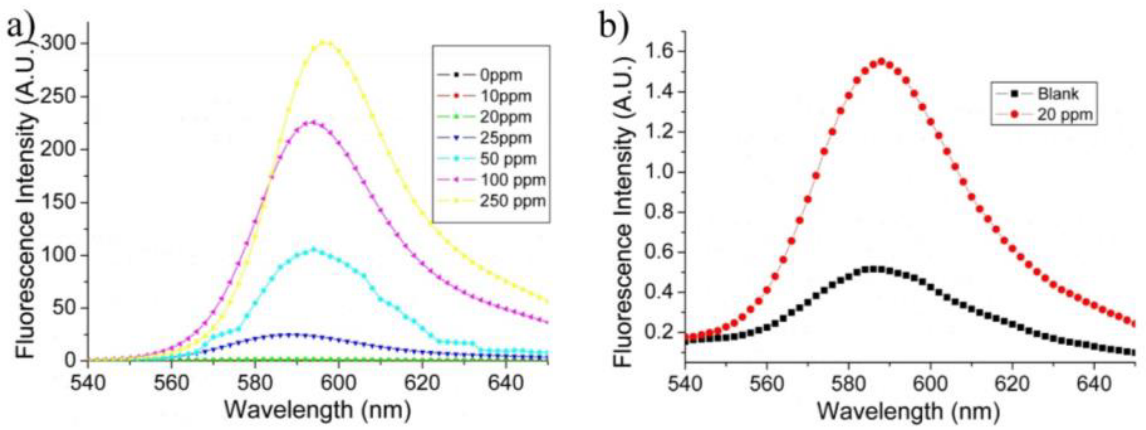

Figure 3. Probe RB-AB (1 mg/mL) detecting DCP in DMF solutions by fluorescence analysis: (a) fluorescence spectra of probe RB-AB reacting with different amounts of DCP (from bottom to top:0, 10, 20, 25, 50, 100 and $250 \mathrm{ppm}$ ) and (b) Probe RB-AB's limit of detection for DCP.

To evaluate RB-AB probe's sensitivity for detection of nerve agents, $\mathrm{DCP}$ at different concentrations was added to RB-AB $(1 \mathrm{mg} / \mathrm{mL})$ in DMF solution. After the detection solutions stayed at RT for 20 minutes, the fluorescence emission spectra were measured. It can be also observed that the fluorescence emission band located at $590 \mathrm{~nm}$ appeared. From figure 3b, the LOD for DCP was identified as $20 \mathrm{ppm}$ under the detection condition as the signal-to-noise ratio is greater than 3 at the amount of $20 \mathrm{ppm}(1.552$ relative to 0.515 in the background). The peak values of fluorescence emission intensity grew as concentrations of DCP changed from 25 to $250 \mathrm{ppm}$ (figure $3 \mathrm{a}$ ).

\subsection{Proposed Mechanisms for RB-AB to Detect Nerve Agent Simulants}

Previously [16], RB-AE (scheme 1b) was used as a probe for detection of DCP in DMF solutions. The structural difference between the molecules of RB-AE and RB-AB is that the two probes contain different reactive groups. RB-AE probe introduces a 2 aminoethanol group to react with the nerve agent simulant while RB-AB uses a 2aminobenzyl alcohol group to do it. The mechanism of RB-AE probe detecting is nucleophilic lactam keto oxygen in its structure attacks on phosphate ester intermediate after reacting with the target object, which results in the lactam-ring opening. Inspired by the result of RB-AE, RB-AB was designed to replace 2-aminoethanol group with 2aminobenzyl alcohol group. Theoretically, the benzyl alcohol group in RB-AB is also an excellent nucleophilic function group that can attack on electrophilic nerve agent molecule to form phosphate ester 5. The formed phosphate ester intermediate of RB-AB could undergo intramolecular transformations to produce detectable colored and fluorescent specie 6 (scheme 3), which is similar to the supposed mechanism of RB-AE for detection. The two different probes working in the similar mechanism emphasized that it is important to explore chemical probes to detecting toxic and dangerous substances through rational molecular design. 


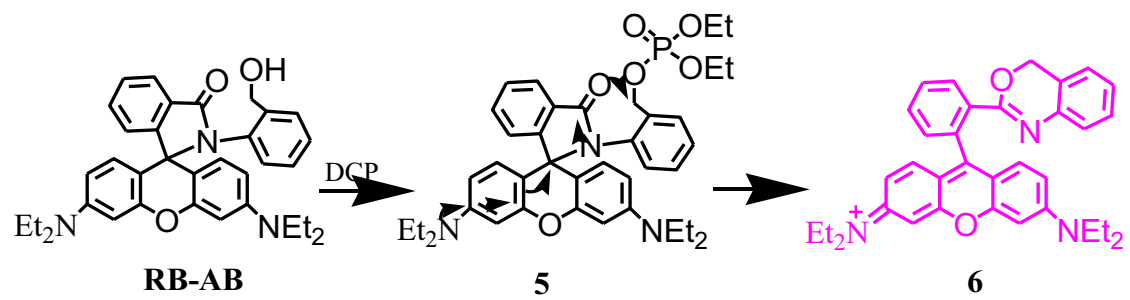

Scheme 3. Proposed mechanisms of RB-AB probe for detection of DCP. Compound 6 is the species with strong fluorescence and obvious color.

\section{Conclusion}

In general, a Rhodamine B-based probe for detecting the simulants nerve agents was reported here. The mechanism for detecting is that 2-aminobenzyl alcohol group of RB$\mathrm{AB}$ reacts with the target molecule rapidly, and the resultant phosphate ester intermediate undergoes the ring-opening of lactam in the probe. The final products in the RB-AB detection solution will exhibit significant fluorescence and color changes, which can be easily recognized by fluorescent or UV-Vis measurements, and even by the naked eye. It is vital importance of rapid and convenient detection of gaseous nerve agents in practical applications, and this paper has showed that our RB-AB probe is capable of rapidly recognizing DCP or DECP at the ppm level. In future, it can be anticipated that through pumping contaminated air into the RB-AB probe solution, the gaseous nerve agent detection could be quickly accomplished with naked eye or a simple fluorescent device during a nerve agent attack.

\section{References}

[1] Sidell F R and Borak J 1992 Chemical warfare agents: II. Nerve agents Ann. Emerg. Med. 21865.

[2] Marrs T C 1993 Organophosphate poisoning Pharmacol. Ther. 5851.

[3] Kim K, Tsay O G, Atwood D A and Churchill D G 2011 Destruction and detection of chemical warfare agents Chemical Reviews 1115345.

[4] Wiener S W and Hoffman R S 2004 Nerve agents: A comprehensive review J. Intensive Care Med. 19 22.

[5] Marrs T C, Rice P and Vale J A 2006 The role of oximes in the treatment of nerve agent poisoning in civilian casualties Toxicol. Rev. 25297.

[6] Xiong W, Gong Y J, Che Y K and Zhao J C 2019 Sensitive discrimination of nerve agent and sulfur mustard simulants using fluorescent coassembled nanofibers with forster resonance energy transferenhanced photostability and emission Analytical Chemistry 911711.

[7] Singh V V 2016 Recent advances in electrochemical sensors for detecting weapons of mass destruction: A review Electroanalysis $\mathbf{2 8} 920$.

[8] Vymazalova K, Halamek E and Kadlcak J 2016 Cholinesterase biosensor for detection of nerve agents Chem. Listy. 110645.

[9] Yan C X, Qi F L, Li S G, Xu J Y, Liu C, Meng Z H, Qiu L L, Xue M, Lu W and Yan Z Q 2016 Functionalized photonic crystal for the sensing of Sarin agents Talanta 159412.

[10] Chai P R, Boyer E W, Al-Nahhas H and Erickson T B 2017 Toxic chemical weapons of assassination and warfare: nerve agents VX and sarin Toxicol. Commun. 121.

[11] Climent E, Biyikal M, Gawlitza K, Dropa T, Urban M, Costero A M, Martinez-Manez R and Rurack K 2017 Determination of the chemical warfare agents Sarin, Soman and Tabun in natural waters employing fluorescent hybrid silica materials Sensors and Actuators B-Chemical 2461056.

[12] Chen L Y, Wu D and Yoon J 2018 Recent advances in the development of chromophore-based chemosensors for nerve agents and phosgene ACS Sensors 327. 
[13] Wu W H, Wang X, Zong L, Li D, Xiao Y H, Sui S H, Li J, Liu M, Chen G Y, Luo T, Liu M, Wang X M and Jiang Z G 2021 A fluorogenic and chromogenic probe distinguishes fluoride anions and thiols: Implications for discrimination of fluoride-containing $\mathrm{G}$ series and sulfur-containing $\mathrm{V}$ series nerve agents J. Fluoresc. 31141.

[14] Dale T J and Rebek J 2006 Fluorescent sensors for organophosphorus nerve agent mimics Journal of the American Chemical Society 1284500.

[15] Burnworth M, Rowan S J and Weder C 2007 Fluorescent sensors for the detection of chemical warfare agents Chemistry-A European Journal 137828.

[16] Wu W H, Dong J J, Wang X, Li J, Sui S H, Chen G Y, Liu J W and Zhang M 2012 Fluorogenic and chromogenic probe for rapid detection of a nerve agent simulant DCP Analyst 1373224.

[17] Lei Z H and Yang Y J 2014 A concise colorimetric and fluorimetric probe for sarin related threats designed via the "covalent-assembly" approach Journal of the American Chemical Society 1366594.

[18] Manna A, Jana K, Guchhait N and Goswami S 2017 Discrimination of tabun mimic diethyl cyanophosphonate from sarin mimic diethyl chlorophosphate via Zn(II)-triggered photoinduced electron transfer-decoupled excited state intramolecular proton transfer processes New Journal of Chemistry $\mathbf{4 1}$ 6661.

[19] Chen L, Oh H, Wu D, Kim M H and Yoon J 2018 An ESIPT fluorescent probe and a nanofiber platform for selective and sensitive detection of a nerve gas mimic Chemical Communications 542276.

[20] Wu W H, Sui S H, Li J, Zong L, Li D, Xiao Y H, Wang L Y, Zhu H Y, Shen Y L and Jiang Z G 2020 A fluorescent probe bearing two reactive groups discriminates between fluoride-containing $\mathrm{G}$ series and sulfur-containing V series nerve agents Analyst 1455425.

[21] So H S, Angupillai S and Son Y A 2016 Prompt liquid-phase visual detection and low-cost vapor-phase detection of DCP: A chemical warfare agent mimic Sensors and Actuators B-Chemical 235447. 